\title{
L(we)-spaces and Some of its Weak Forms
}

\author{
${ }^{1}$ Nadia A. Nadhim \\ ${ }^{1}$ University of AL-Anbar \\ Faculty of Education for \\ pure sciences
}

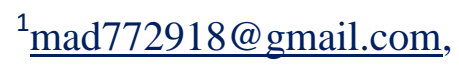

Recived : $23 \backslash 1 \backslash 2019$
${ }^{2}$ Haider J. Ali

${ }^{2}$ University of AL-

Mustansiriyah

College of Science

²haiderali89@yahoo.com, 3 rashanm6@gmail.com

\author{
${ }^{3}$ Rasha N. Majeed \\ ${ }^{3}$ University of Baghdad \\ Faculty of Education for pure \\ sciences Abn AL-Haitham
}

Available online : $23 / 4 / 2019$

\begin{abstract}
:
In this paper, we provide a new generalization of $\mathrm{L} \mathcal{C}$-spaces which is $\mathrm{L}(w \mathcal{C})$-spaces, $w \mathrm{~L}(w \mathcal{C})$-spaces also another weak forms of $\mathrm{L}(w \mathcal{C})$-spaces which is called $w \mathrm{~L}_{i}$-spaces, $(i=1,2,3,4)$. In addition, we give the relationships between these new types and studied the heredity property for each type.
\end{abstract}

MSC. 54F05,54E55,54D10 .

Keywords:

Lindelof space, $L \mathcal{C}_{\text {-space, }}$ closed set, $w_{\text {-closed set, }} w_{\text {-continuous function. }}$ 


\section{1-Introduction}

The concept of Lindelof space was introduced in 1929 by Alexandroof and Urysohn, since there is no relation between Lindelof space and closed sets, so this point stimulated some researchers to introduce a new concept namely $L \mathcal{C}_{\text {-space. }}$

The notion of $L \mathcal{C}_{\text {-space was first introduced in }}$ 1979 by Mukherji and Sarkar [15], that is "Every Lindelof subsets are closed", some authors name it L-closed space [7], [14] and [18]. LC $\mathcal{C}_{\text {-space }}$ different from Lindelof space and there is no relation between Lindelof and $L \mathcal{C}_{\text {-space. }}$

Near closed sets has an important role in topological spaces as a generalized of closed sets. Hdeib in 1982 [10] introduce the concept of $w_{-}$ closed set that is " A subset $\mathcal{N}$ is called $w$-closed, if $\mathcal{N}$ contains all its condensation points.The family of all $\mathcal{W}$-open subset of a space $X$ denoted by $T_{w}$ forms a topology on $X$ which is finer than $T$, several characterization and facts of $\mathcal{W}$-closed subset where provided in [3], [1] and [11].

We introduce in this work a new concept which

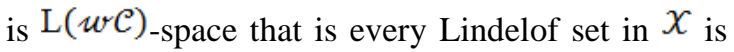
$w_{\text {-closed, and }} \mathrm{L}(w \mathcal{C})_{\text {-space }}$ is a generalized of

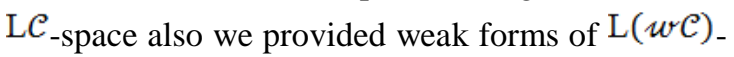
space namely $w \mathrm{~L}_{i \text {-space }} i=1,2,3,4$, and introduce the relationships between themselves also with $\mathrm{L}(w \mathcal{C})$-space and then define $w \mathrm{~L}(w \mathcal{C})$. space which is weaker form of $\mathrm{L}(w \mathcal{C})$-space, several properties and theorem which link between those concepts. Finally, we give several facts and examples to support this concepts.

\section{2-Preliminaries}

Definition 2.1 [10] Let $\mathcal{N}$ be a subset of a space $X$, a point $e \in X$ is called condensation point of $\mathcal{N}$, if for any open set $\mathcal{V}$ and $e \in \mathcal{V}$, the set $\mathcal{V} \cap \mathcal{N}$ is uncountable, if $\mathcal{N}$ contains all its condensation points then it is $w$-closed.

Definition 2.2 [11] A subset $U$ of a space $X$ is said

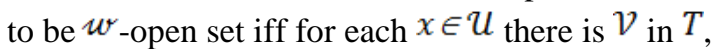
$x \in \mathcal{V}$ and $\mathcal{V}-\mathcal{U}$ is countable.

\section{Nadia .A/Haider .J/Rasha .N}

Remark 2.3 [16] Every closed ( resp., open) set is

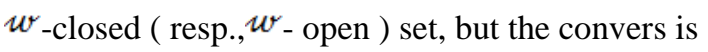
not true.

Example 2.4 Let ( $\mathcal{R}, T_{\text {ind }}$ ) be indiscrete space on a real line $\mathcal{R}$, the set of all irrational numbers $\mathbb{Q}^{C}$ which is subset of $\mathcal{R} \mathcal{R}, \mathbb{Q}^{C}$ is $\mathcal{W}_{\text {- }}$ open set but not open. Also the rational numbers $\mathbb{Q}$ is $w$-closed but not closed.

Definition 2.5 [6] A subset $\mathcal{N}$ of a space $X$ is said to be clopen set if it is open and closed in $X$.

Definition 2.6 [10] Let $\left(X, T_{w}\right)$ be a topological space and $\mathcal{A}$ be a subset of $X$, then

1.

2.

Proposition 2.7 [9] If $(X, T)$ be a topological space. $\mathcal{A}, \mathcal{B}$ subsets of $\mathcal{X}$, then

1. is an $w$-open subset of $X$.

2.

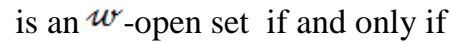
$\mathcal{A}=\operatorname{Int}_{w}(\mathcal{A})$.

3.

$$
\mathcal{A} \text { and } \operatorname{Int}(\mathcal{A}) \subseteq \operatorname{Int}_{w}(\mathcal{A}) .
$$

4. is an $w_{\text {-closed set. }}$

5. is an $w_{\text {-closed set if and only }}$ if $\mathcal{A}=C l_{w}(\mathcal{A})$.

6.

$$
C l_{w}(\mathcal{A}) \text { and } C l_{w}(\mathcal{A}) \subseteq C l(\mathcal{A})
$$


Definition 2.8 [16] A subset $\mathcal{A}$ of a space $X$ is said to be an $\mathcal{W}_{\text {-set if }} \mathcal{A}=U \cap \mathcal{V}$, where

$\operatorname{Int}(\mathcal{V})=\operatorname{Int}_{\mathcal{W}}(\mathcal{V})$ and $U$ is open set.

Remark 2.9 [16] Let $\mathcal{N}$ be a subset of a space $x$, then $\mathcal{N}$ is open if and only if $\mathcal{N}$ is $w_{\text {-open and }} w_{\text {- }}$ set.

Example 2.10 Let $\left(\mathcal{R}, T_{U}\right)$ be a usual space on a real line $\mathcal{R}$, the set of all irrational numbers $\mathbb{Q}^{C}$ is

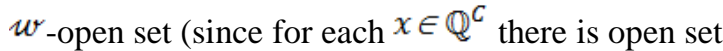
$U$ subset of $\mathcal{R}$ containing $x$ such that $U-\mathbb{Q}^{C}$ is countable such as $\sqrt{2} \in \mathbb{Q}^{C}$ and $(0,2) \subseteq \mathcal{R}$ and $\sqrt{2} \in(0,2)$ and $(0,2)-\mathbb{Q}^{C}=\mathbb{Q}$ is countable), but $\mathbb{Q}^{C}$ is not $\mathcal{W}_{\text {-set since }} \mathbb{Q}^{C}=\mathcal{R} \cap \mathbb{Q}^{C}$, where $\mathcal{R}$ is open set but $\operatorname{Int}\left(\mathbb{Q}^{C}\right)=\emptyset \neq \mathbb{Q}^{C}=\operatorname{Int}_{w}\left(\mathbb{Q}^{C}\right)$,

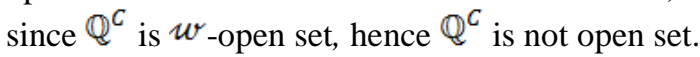

Proposition 2.11 [20] If $\mathcal{N}$ is an $w_{\text {-closed (resp., }}$ $w$-open) subset of $X$ and $\mathcal{A} \subseteq X$ then $\mathcal{A} \cap \mathcal{N}$ is an

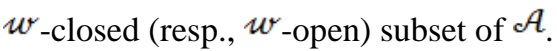

Proposition 2.12 [20] Let ${ }^{\mathcal{P}}$ be an $w_{\text {-closed (resp., }}$ $w$-open) of a space $X$. If $\mathcal{N}$ is $w_{\text {-closed (resp., }}$

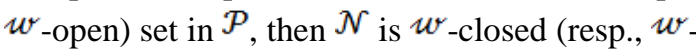
open) set in $X$. Remark 2.13 [20] If $X$ is a space and $\mathcal{G}$ is a subspace of $\mathcal{X}$ such that $\mathcal{B} \subseteq \mathcal{G}$ and $\mathcal{B}$ is $w$-closed (resp., $w$-open) subset in $X$. Then $\mathcal{B}$ is $w$-closed (resp., $w$-open) set in $\mathcal{G}$.

Definition 2.14 [3] A space $X$ is called anti-locally countable space if each open set is an uncountable set.

Proposition 2.15[16] If a space $X_{\text {is anti-locally }}$ countable space then:

1. $(\mathcal{S})$, for any $w_{\text {-closed set }} \mathcal{S}$ in $X$.

2.

$$
(\mathcal{N}) \text {, for any } w_{\text {-open set }} \mathcal{N} \text { in } x \text {. }
$$

Definition 2.16 [19] A space $X$ is said to be $w T_{1-}$ space if for any $, b \in X, a \neq b$, there exists $w_{\text {- }}$ open sets $\mathcal{N}, \mathcal{M}$ with $a \in \mathcal{N}, b \notin \mathcal{N}_{\text {and }}$ $b \in \mathcal{M}, a \notin \mathcal{M}$.
Nadia .A/Haider .J/Rasha .N

Proposition 2.17 [19] A space $X$ is $w T_{1 \text {-space iff }}$ any singleton set is $w$-closed.

Definition 2.18 [19] A space $X$ is said to be $w_{-} T_{2-}$ space if for any two points $x, y$ of $X$, with $x \neq y$

there exist $w_{\text {-open sets }} U, \mathcal{V}$ and $x \in \mathcal{U}, y \in \mathcal{V}$, such that $u \cap \mathcal{v}=\emptyset$.

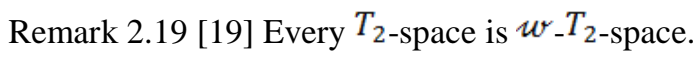

The next example refers to the invers direction of Remark 2.17 not hold:

Example 2.20 If $\mathcal{X}$ is a finite set contain more than one point and ${ }^{T}$ be a discrete topology defined on $X$, then $X_{\text {is }} w_{-} T_{2-\text { space but not }} T_{2 \text {-space. }}$

Proposition 2.21 [13] Let $X$ be an anti-locally countable space then $X$ is an $w_{-} T_{2}$-space iff $X$ is $T_{2 \text {-space. }}$

Definition 2.22 [6] Let $U$ be a collection of a subset of a space $X, U$ is called open cover of $X$, if $U$ is cover $X$ and $U$ is a subfamily of a topology $T$.

Definition 2.23 [6] A space $X$ is said to be Lindelof space if for any open cover of $X$, there is a countable sub cover.

Example 2.24 A countable topological space is a Lindelof space.

Proposition 2.25 [10] In a Lindelof space every closed subset is Lindelof subset.

Proposition 2.26 [17] If $\mathcal{A}$ is Lindelof in $X$ and $\mathcal{B}$ is $w_{\text {-closed in }} X$ then $\mathcal{A} \cap \mathcal{B}$ is Lindelof in $X$.

Definition/2t(2) [ 4] IAtspace $X$ is $w$-Lindelof space, if for each $w$-open cover of $X$ has a countable sub cover.

$$
C l(\mathcal{N})=C l_{w}
$$

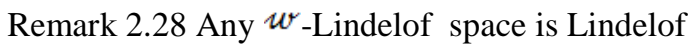
space.

Proposition 2.29 [10] In Lindelof space, every $w_{\text {- }}$ closed set is Lindelof set. 
Definition $2.30[7,15]$ A space $\mathcal{X}$ is said to be $\mathrm{L} \mathcal{C}$. space if any Lindelof set in $X$ is closed.

Example 2.31 A discrete space on a non-empty set $\chi,\left(X, T_{D}\right)$ is $\mathrm{L} \mathcal{C}_{\text {-space. }}$

Example 2.32 Let $\left(\mathcal{R}, T_{\text {Cof }}\right)$ be a co-finite topology on a real line $\mathcal{R}$, the set of rational numbers $\mathbb{Q}$ is Lindelof, not closed, so $\left({ }^{\mathcal{R}, T_{\text {Cof }}}\right)$ is not $\mathrm{LC}_{\text {-space. }}$

Proposition 2.33 [2] Let $f:(\mathcal{X}, T) \rightarrow\left(\mathcal{Y}, T^{\prime}\right)$ be a bijective open function, if $X$ is $L \mathcal{C}_{\text {-space then }} \mathcal{Y}$ is $\mathrm{L} \mathcal{C}_{\text {-space. }}$

Definition 2.34 [8] A space $X$ is said to be Locally $\mathrm{L} \mathcal{C}_{\text {-space if any point has a neighborhood }}$ which is an $L \mathcal{C}_{\text {-subspace. }}$

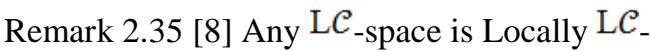
space

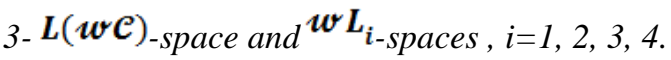

In this section, we define $\mathrm{L}(w \mathcal{C})$-space and state four weaker forms of $\mathrm{L}(w \mathcal{C})$-space also we study their relationship with $\mathrm{L} \mathcal{C}_{\text {-space. }}$

Definition 3.1 A space $X$ is called $\mathrm{L}(w \mathcal{C})_{\text {-space if }}$ for each Lindelof set in $X$ is $w$-closed.

Example 3.2 The integer numbers $\boldsymbol{Z}$ defined on a topology $T$ as follows: $T_{E x c=\{} U \subseteq Z, x_{0} \notin U$, for some $\left.x_{0} \in Z\right\} \cup\{Z\}$ be excluded point topology on $z$, let $x_{0}=6$, so $z-\{6\} \subseteq Z$ is countable, so it

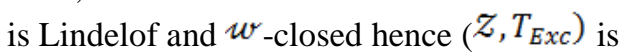
$\mathrm{L}(w \mathcal{C})_{\text {-space. }}$

Remark 3.3 Every $L \mathcal{C}_{\text {-space is }} \mathrm{L}(w \mathcal{C})$-space.

The following example show that the convers of Remark 3.3 is not hold.

Example 3.4 Let ( $\left(\mathcal{R}, T_{\text {Cof }}\right)$ be a co-finite topology on a real line $\mathcal{R},\left(\mathcal{R}, T_{\text {Cof }}\right)$ is $\mathrm{L}(w \mathcal{C})$-space but not $L \mathcal{C}_{\text {-space. }}$

Proposition 3.5 Every subspace of $\mathrm{L}(w \mathcal{C})_{\text {-space is }}$ $\mathrm{L}(w \mathcal{C})_{\text {-space. }}$
Proof: Suppose a space $\mathcal{X}$ is $\mathrm{L}(w \mathcal{C})$ and $\mathcal{G}$ be subspace of $\mathcal{X}$, a subset $\mathcal{M}$ is a Lindelof in $\mathcal{G}$, so $\mathcal{M}$ is Lindelof in $X$ and then $\mathcal{M}$ is $w$-closed in $X$, from Proposition 2.13, we get $\mathcal{M}$ is $w$-closed in $\mathcal{G}$, hence $\mathcal{G}$ is $\mathrm{L}(w \mathcal{C})$-space

Proposition 3.6 Every $\mathrm{L}(w \mathcal{C})$-space is $w T_{1 \text {-space. }}$

Proof: Let $e \in \mathcal{X}$, then $\{e\}$ is Lindelof subset in $X$, but $X$ is $\mathrm{L}(w \mathcal{C})$-space, so $\{e\}$ is $w$-closed from Proposition $2.17, X$ is $w T_{1-\text { space. }}$

Definition 3.7 A subset $\mathcal{F}$ of a space $\mathcal{X}$ is said to be $\mathcal{F}_{\mathrm{e}-} w_{\text {-closed set if }} \mathcal{F}$ is the union of countable $w_{\text {- }}$ closed sets.

Definition 3.8 A subset $G$ of a space $X$ is said to be $\mathcal{S}_{\partial-} w_{\text {-open set, if }} G$ is the intersection of countably $w_{\text {-open sets. }}$

Remark 3.9

1.

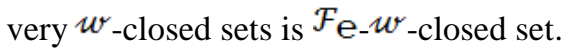

2. very $w_{\text {-open sets is }} \mathbb{S}_{\partial-} w_{\text {-open set. }}$

Example 3.10 Let $\left(\mathcal{R}, T_{\text {Cof }}\right.$ ) be a co-finite topology on a real line $\mathcal{R}$, a natural numbers $\mathcal{N}$ of $\mathcal{R}$ is not

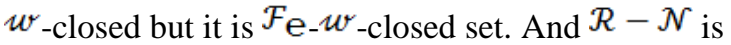

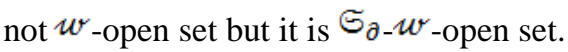

Definition 3.11 [8] A topological space $(X, T)$ is said to be $\mathcal{P}^{\star}$-space if any $\mathcal{S}_{\partial-} w_{\text {-open subset of }} \mathcal{X}$ is $w$-open set.

Definition 3.12 A space $X$ is said to be:

1. -space if any Lindelof $\mathcal{F}_{\mathrm{e}-w_{\text {-closed set is }}}$ $w_{\text {-closed set. }}$

2. $w \mathrm{~L}_{2 \text {-space if }} \mathcal{N}$ is Lindelof in $X$, then. $C l_{w}(\mathcal{N})$ is Lindelof.

3. $w \mathrm{~L}_{3 \text {-space if for each Lindelof subset }} \mathcal{N}$ is $\mathcal{F}_{\text {e- }} w_{\text {-closed set. }}$ 
4.

$$
\begin{aligned}
& w \mathrm{~L}_{4-\text {-space if }} \mathcal{N} \text { is a Lindelof in a space } \\
& X \text { then there is a Lindelof } \mathcal{F}_{\mathrm{e}-w} \text {-closed } \\
& \text { set } \mathcal{M} \text { and } \mathcal{N} \subseteq \mathcal{M} \subseteq C l_{w}(\mathcal{N}) .
\end{aligned}
$$

Example 3.13 The usual topology define on the set of real number $\mathcal{R},\left(\mathcal{R}, T_{U}\right)$ is an $w \mathrm{~L}_{2 \text {-space but }}$

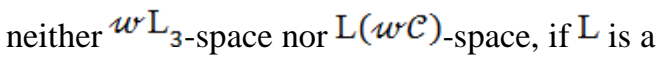
Lindelof set in $\mathcal{R}$, since $\mathcal{R}$ is second countable space, then $C l_{w}(\mathrm{~L})$ is second countable subspace, then $C l_{w}(\mathrm{~L})$ is a Lindelof, so $\mathcal{R}$ is an $w \mathrm{~L}_{2 \text {-space }}$

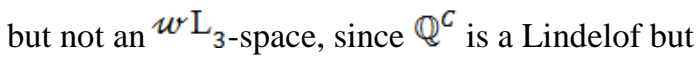

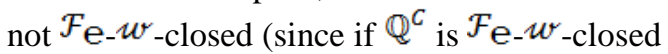
then $\mathbb{Q}$ is $\mathcal{S}_{\partial-} w_{\text {-open set that is }} \mathbb{Q}=\bigcap_{i=1}^{\infty} G_{i}$ where $G_{i}$ is an $w_{\text {-open set in }} \mathcal{R}$, so $\mathbb{Q} \subseteq G_{i}$ for each $\mathrm{i}$, but the only $w_{\text {-open set containing }} \mathbb{Q}$ is $\mathcal{R}$, that is $G_{i}=\mathcal{R}$ for each i and $\mathbb{Q}=\bigcap_{i=1}^{\infty} G_{i}=\mathrm{R}$ which is contradiction, also $\mathcal{R}$ is not $\mathrm{L}(w \mathcal{C})$-space (since $\mathbb{Q}^{C}$ is a Lindelof but not $w_{\text {-closed). }}$

Proposition 3.14 Every $\mathrm{L}(w \mathcal{C})$-space is $w \mathrm{~L}_{1}($ resp., $w \mathrm{~L}_{2}, w \mathrm{~L}_{3}, w \mathrm{~L}_{3}$ )-space.

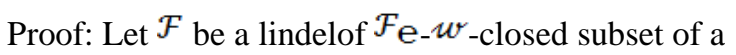
space $X$, since $X$ is an $\mathrm{L}(w \mathcal{C})_{\text {-space, so }} \mathcal{F}$ is $w_{\text {- }}$ closed set, hence $X$ is $w \mathrm{~L}_{1 \text {.Now, let }} \mathcal{A}$ be a lindelof in $X$,but $X$ is $\mathrm{L}(w \mathcal{C})_{\text {-space, so }} \mathcal{A}$ is $w$. closed and by Proposition 2.7 part (5), $C l_{w}(\mathcal{A})=\mathcal{A}$, hence $C l_{w}(\mathcal{A})$ is Lindelof and then $X$ is $w \mathrm{~L}_{2}$. Let $\mathcal{B}$ be a lindelof set of $\mathcal{X}$, since $X$ is $\mathrm{L}(w \mathcal{C})$-space, then $\mathcal{B}$ is $w$-closed, so $\mathcal{B}$ is

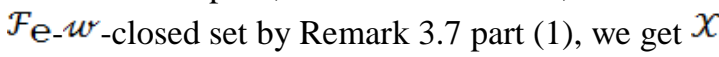
is $w \mathrm{~L}_{3}$. Let $\mathcal{D}$ be a lindelod in $X$, also $X$ is $\mathrm{L}(w \mathcal{C})$-space, then $\mathcal{D}$ is $w$-closed, from Remark 3.9 part (1), $\mathcal{D}$ is $\mathcal{F}_{\mathrm{e}-} w_{\text {-closed set, put }} \mathcal{D}=\mathrm{L}$, then $\mathcal{D} \subseteq \mathrm{L} \subseteq C l_{w}(\mathcal{D})$, so $X$ is an $w \mathrm{~L}_{4-\text { space }}$

Proposition 3.15 If a space $X$ is $w \mathrm{~L}_{1}$ and $w \mathrm{~L}_{3-}$ spaces then it is $\mathrm{L}(w \mathcal{C})_{\text {-space. }}$

Proof: Let $\mathcal{K}$ be a Lindelof of $\mathcal{X}$, since $X$ is $w \mathrm{~L}_{3-}$ space, so $\mathcal{K}_{\text {is }} \mathcal{F}_{\mathrm{e}-w_{\text {-closed, also }}} \mathcal{X}_{\text {is an }} w \mathrm{~L}_{1-}$ space, hence $\mathcal{K}$ is $w$-closed that is $X$ is $\mathrm{L}(w \mathcal{C})$. space.

Proposition 3.16 If a space $X$ is $w \mathrm{~L}_{1}$ and $w \mathrm{~L}_{4-}$ spaces then it is $w \mathrm{~L}_{2 \text {-space. }}$

Proof: Let $X_{\text {be an }}{ }^{w L_{4}}$-space, $\mathcal{N}_{\text {be a Lindelof in }}$ $\chi$, so there exists a Lindelof $\mathcal{F}_{\text {e- }} w_{\text {-closed }} \mathcal{S}$ with

$\mathcal{N} \subseteq \mathcal{S} \subseteq C l_{w}(\mathcal{N})$, since $X$ is $w \mathrm{~L}_{1-\text { space, we }}$ get $\mathcal{S}$ is $w$-closed set and $\mathcal{S}=C l_{w}(\mathcal{S})$ by

Proposition 2.7 part (5), but $\mathcal{N} \subseteq \mathcal{S}$ then $C l_{w}(\mathcal{N}) \subseteq$ $C l_{w}(\mathcal{S})=\delta$, so $C l_{w}(\mathcal{N})=\delta$, and since $\delta$ is

Lindelof then $C l_{w}(\mathcal{N})$ is Lindelof, therefore $X$ is $w \mathrm{~L}_{2 \text {-space. }}$

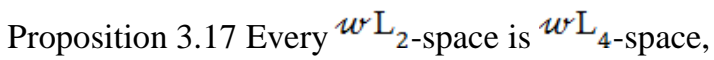

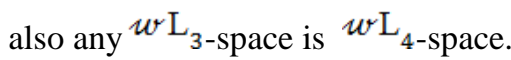

Proof: Let $G$ be a Lindelof in a space $X$, since $X$ is $w \mathrm{~L}_{2}$, so $C l_{w}(G)$ is Lindelof and $w$-closed set then $C l_{w}(G)$ is $\mathcal{F}_{\mathrm{e}-w}$-closed and $G \subseteq C l_{w}(G)$ $\subseteq C l_{w}(G)$, take $\mathcal{F}=C l_{w}(G)$, then $G \subseteq \mathcal{F}_{\subseteq} C l_{w}(G)$ that is $X$ is $w \mathrm{~L}_{4 \text {-space. To prove }}$ the second part let $\mathcal{J}$ be a Lindelof set of a space

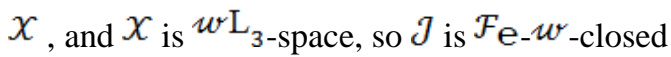
subset in $X$, take $\mathcal{J}=\mathcal{N}$ and $\mathcal{J} \subseteq \mathcal{J} \subseteq C l_{w}(\mathcal{J})$, hence $\mathcal{J} \subseteq \mathcal{N} \subseteq C l_{w}(\mathcal{J})$, hence $X$ is $w \mathrm{~L}_{4 \text {-space. }}$

Proposition 3.18 Every $\mathcal{P}^{*}$-space is an ${ }^{w} \mathrm{~L}_{1-\text { space. }}$

Proof: Let $G$ be a Lindelof $\mathcal{F}_{\mathrm{e}-} w_{\text {-closed set of }} X$, but $X$ is $\mathcal{P}^{*}$-space and $G^{c}$ is $\mathcal{S}_{\partial-} w_{\text {-open set of }} \mathcal{X}$, so $G$ is $w$-closed, therefore $X$ is $w L_{1-\text { space. }}$

Definition 3.19 A subset ${ }^{\mathcal{M}}$ of a space $\mathcal{X}$ is said to be $w_{\text {-dense if }} \mathrm{Cl}_{w}(\mathcal{M})=\chi$.

Proposition 3.20 Each Lindelof space is an $w \mathrm{~L}_{2-}$

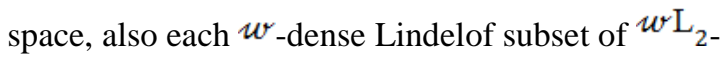
space is Lindelof.

Proof: Let ${ }^{\mathcal{P}}$ be a Lindelof set in $X, C l_{w}(\mathcal{P})$ is $w$. closed in $X$ from Proposition $2.29, C l_{w}(\mathcal{P})$ is 
Lindelof, then $X$ is an $w \mathrm{~L}_{2 \text {-space. Now, let } \mathcal{F}}$ be $w$-dense Lindelof in $X$, hence $C l_{w}(\mathcal{F})=X$

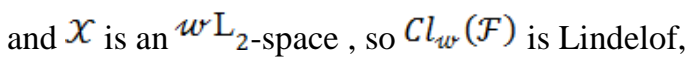
that is $X$ Lindelof.

Theorem 3.21 The property $w \mathrm{~L}_{3 \text {-space is }}$ hereditary property.

Proof: Let $\mathcal{A}$ be a subspace of $w \mathrm{~L}_{3 \text {-space }} \mathcal{X}$, and $\mathrm{L}$ is a Lindelof subset of $\mathcal{A}$, then $\delta_{\text {is Lindelof }}$ subset of $\mathcal{X}$, by hypothesis $\mathcal{S}_{\text {is }} \mathcal{F}_{\text {e- }} w_{\text {-closed, so }}$ there is $\left\{\mathcal{M}_{n}\right\}_{n \in I}$ a family of $w_{\text {-closed in }} X$ and $\mathcal{S}=\mathrm{U}_{n \in I} \mathcal{M}_{n}$, take $\mathcal{M}_{n=}^{*} \mathcal{M}_{n} \cap \mathcal{A}$, so $\mathcal{M}_{n}^{*}$ is $w_{\text {- }}$ closed sets in $\mathcal{A}$, for each $n$, also $s=s \cap \mathcal{A}=\left(\mathrm{U}_{n \in I} \mathcal{M}_{n}\right) \cap \mathcal{A}=\mathrm{U}_{n \in I}\left(\mathcal{M}_{n}\right.$ $\cap \mathcal{A})=\mathrm{U}_{n \in I} \mathcal{F}_{n}^{*}$, so $\mathcal{S}_{\text {is }} \mathcal{F}_{\text {e- }} w_{\text {-closed in }} \mathcal{A}$, hence $\mathcal{A}$ is an $w \mathrm{~L}_{3 \text {-space. }}$

Theorem 3.22 Each $\mathcal{F}_{\text {e- }} w$-closed subset of $w \mathrm{~L}_{1}$ ( resp., $w \mathrm{~L}_{2}, w \mathrm{~L}_{4}$ )-space is $w \mathrm{~L}_{1}$ ( resp., $w \mathrm{~L}_{2}$, $\left.w \mathrm{~L}_{4}\right)$-space.

Proof: Let $X$ be $w \mathrm{~L}_{1 \text {-space, }} \mathcal{A}_{\text {be }} \mathcal{F}_{\mathrm{e}-w_{\text {-closed }}}$

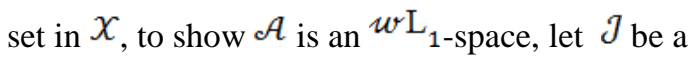
Lindelof $\mathcal{F}_{\mathrm{e}-w}$-closed set in $\mathcal{A}$ that is there exist a collection $\left\{\mathcal{S}_{i}^{*}\right\}_{i \in J}$ of $w_{\text {-closed sets in } \mathcal{A}_{\text {with }}}$ $\mathcal{J}=\bigcup_{i \in J} s_{i}^{*}$, let $\mathcal{S}_{i}^{*}=\delta_{i} \cap \mathcal{A}$, whenever $\delta_{i}$ is an $w_{\text {-closed in }} X_{\text {for all i, so }}$ $\mathcal{J}=\mathrm{U}_{i \in J}\left(\mathcal{A} \cap s_{i}\right)=\mathcal{A} \cap\left(\mathrm{U}_{i \in J} s_{i}\right)=$ $\left(\mathrm{U}_{j \in I} \mathcal{V}_{j}\right) \cap\left(\mathrm{U}_{i \in J} \mathcal{S}_{i}\right)$, where $\mathcal{V}_{j}$ is an $w_{\text {-closed }}$ subset of $\mathcal{X}$, since $\mathcal{A}$ is an $\mathcal{F}_{\text {e- }} w_{\text {-closed, so }}$ $\mathcal{J}=\mathrm{U}_{i, j \in J}\left(\mathcal{S}_{i} \cap \mathcal{V}_{j}\right)$, L is a Lindelof $\mathcal{F}_{\mathrm{e}-w_{\text {-closed }}}$ closed in $\mathcal{A}$ therefore $\mathcal{A}$ is $w \mathrm{~L}_{1 \text {-space . To show }}$

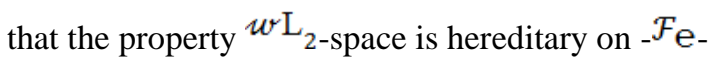
$w_{\text {-closed, let }} \mathcal{K}$ be a Lindelof in $\mathcal{A}$, hence $\mathcal{K}$ is Lindelof in $X$, also $X$ is $w \mathrm{~L}_{2-\text { space, so }} C l_{w}(\mathcal{K})$ Lindelof ,

$C l_{w \text { in } \mathcal{A}}(\mathcal{K})=$ $C l_{w \text { in } x}(\mathcal{K}) \cap \mathcal{A}=C l_{w \text { in } X}(\mathcal{K}) \cap\left(\bigcup_{i \in I} \mathcal{F}_{i}\right)$, where $\mathcal{F}_{i}$ is an $w_{\text {-closed in }} \mathcal{A}_{\text {which is }} \mathcal{F}_{\text {e- }} w_{\text {- }}$ closed, $C l_{w \text { in } \mathcal{A}}(\mathcal{K})=U_{i \in I}\left(C l_{w \text { in } x}(\mathcal{K}) \cap \mathcal{F}_{i}\right)$ is Lindelof ( since a countable union of Lindelof subset is Lindelof ), then $C l_{w \text { in }} \mathcal{A}(\mathcal{K})$ is Lindelof,

\section{Nadia .A/Haider .J/Rasha .N}

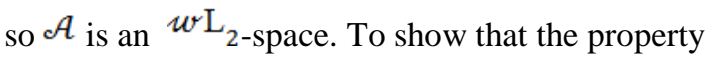

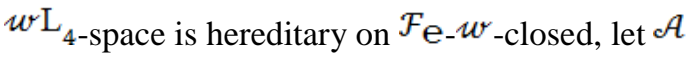
be $\mathcal{F}_{\mathrm{e}-} w_{\text {-closed in }} w \mathrm{~L}_{4 \text {-space }} \mathcal{X}$, to show $\mathcal{A}$

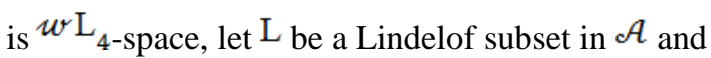
hence it is Lindelof in $X$, so there is a Lindelof $\mathcal{F}_{\mathrm{e}}$ $w_{\text {-closed }} \mathcal{F}$ in $X$ with $\mathrm{L} \subseteq \mathcal{F} \subseteq C l_{w \text { in } x}(\mathrm{~L})$, put $\mathcal{H}=\mathcal{F} \cap \mathcal{A}$ and $\mathcal{A}=\mathrm{U}_{i \in I} \mathcal{C}_{i}$, where $\mathcal{C}_{i}$ is $w_{\text {- }}$ closed in $\mathcal{A}$, to show $\mathcal{H}$ is a Lindelof $\mathcal{F}_{\mathrm{e}-w_{\text {- }}}$ closed in $\mathcal{A}$ since $\mathcal{H}=\mathcal{F} \cap \mathcal{A}=\mathcal{F} \cap\left(\mathrm{U}_{i \in I} \mathcal{C}_{i}\right)=\mathrm{U}_{i \in I}\left(\mathcal{F} \cap \mathcal{C}_{i}\right)$ and $\left(\mathcal{F} \cap \mathcal{C}_{i}\right)$ is $w_{\text {-closed in }} \mathcal{F}$ which is Lindelof, then for all $\mathrm{i},\left(\mathcal{F} \cap \mathcal{C}_{i}\right)$ is Lindelof and so $\mathcal{H}=\mathrm{U}_{i \in I}\left(\mathcal{F} \cap \mathcal{C}_{i}\right)$ is Lindelof ( since a countable

union of Lindelof subset is Lindelof), to show $\mathcal{H}$ is $\mathcal{F}_{\text {e- }} w_{\text {-closed }} \mathcal{F}$ in $\mathcal{A}$, let $\mathcal{F}=\mathrm{U}_{j \in I} \mathcal{N}_{j}$, where $\mathcal{N}_{j}$

is an $w_{\text {-closed in }} \mathcal{X}$, so $\mathcal{H}=\mathcal{F} \cap \mathcal{A}=\left(\mathrm{U}_{j \in I} \mathcal{N}_{j}\right) \cap\left(\mathrm{U}_{i \in I} \mathcal{C}_{i}\right)=$ $\mathrm{U}_{i, j \in I}\left(\mathcal{N}_{j} \cap \mathcal{C}_{i}\right)=\mathrm{U}_{n \in I} s_{n}$

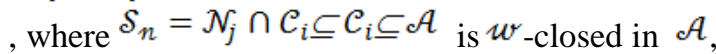

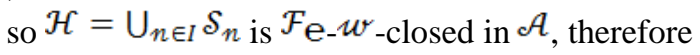
$\mathrm{L} \subseteq \mathcal{H} \subseteq C l_{w \text { in }}(\mathrm{L})$, so $\mathcal{A}$ is $w \mathrm{~L}_{4 \text {-space. }}$

Proposition 3.23 Let $(X, T)$ be an $\mathcal{W}$-Hausdorff

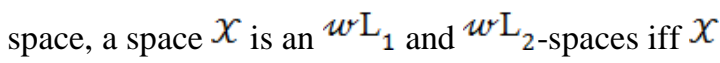
is an is $\mathrm{L}(w \mathcal{C})_{\text {-space. }}$

Proof: Let $\mathcal{K}$ be a Lindelof in $\mathcal{X}$, let $x \notin \mathcal{K}$, so for any $y \in \mathcal{K}$ there exists an $w_{\text {-open sets }} \mathcal{V}_{y}$ containing y, with $x \in C l_{w}\left(\mathcal{V}_{y}\right)$, now $\left\{\mathcal{V}_{y}: \mathrm{y} \in \mathcal{K}\right\}$ is cover of $\mathcal{K}$ and $\mathcal{K}$ is Lindelof, so there exists a countable set $\mathcal{C} \subseteq \mathcal{K}$ such that $\mathcal{K} \subseteq \mathrm{U}\left\{\mathcal{V}_{y}: \mathrm{y} \in \mathcal{C}\right\} \subseteq \mathrm{U}\left\{C l_{w}\left(\mathcal{V}_{y}\right): \mathrm{y} \in \mathcal{C}\right\}$, for each $\mathrm{y} \in \mathcal{C}, \mathcal{K} \cap C l_{w}\left(\mathcal{V}_{y}\right)$ is Lindelof by Proposition 2.23 and $C l_{w}\left(\mathcal{K} \cap C l_{w}\left(\mathcal{V}_{y}\right)\right)$ is

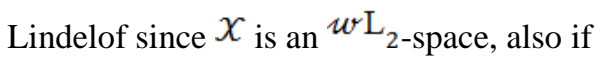
$\mathcal{N}=\mathrm{U}\left\{C l_{w}\left(\mathcal{K} \cap C l_{w}\left(\mathcal{V}_{y}\right)\right): \mathrm{y} \in \mathcal{C}\right\}$, then $\mathcal{N}$ is Lindelof $\mathcal{F}_{\mathrm{e}-w_{\text {-closed and since }}} X$ is $w \mathrm{~L}_{1 \text {-space, }}$

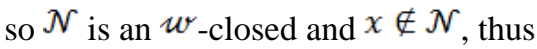
$x \notin C l_{w}(\mathcal{K})$, this show that $\mathcal{K}$ is $w_{\text {-closed subset }}$ of $X$.

4-Locally $\boldsymbol{L}(\boldsymbol{w C})_{- \text {-space }}$ 
In this part we define Locally $\mathrm{L}(w \mathcal{C})$-space, and some theorems, properties about Locally $\mathrm{L}(w \mathcal{C})$. space which is generalized of $\mathrm{L}(w \mathcal{C})_{\text {-space. }}$

Definition 4.1 A space $X$ is said to be Locally

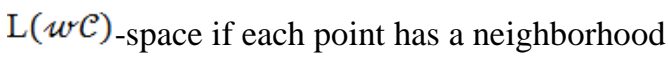
which is an $\mathrm{L}(w \mathcal{C})$-subspace.

Clearly any Locally $\mathrm{L} \mathcal{C}_{\text {-space is Locally }} \mathrm{L}(w \mathcal{C})$ space.The following example refers the invers direction is not hold.

Example 4.2 Let $\left(\mathcal{Z}, T_{E x c}\right)$ be an Excluded point topology on the integer numbers $\boldsymbol{Z}$, where $T_{E x c}=\left\{U \subseteq X, x_{0} \notin U\right.$, for some $\left.x_{0} \in X\right\} \cup\{X\}$. Let $x_{0}=6$, since for each $y \in Z$, since $\{y\}$ is finite, so $\{y\}$ is countable and then it is Lindelof in

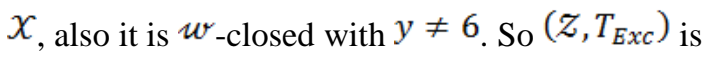
Locally $\mathrm{L}(w \mathcal{C})$-space, but $\{y\}$ is not closed, hence this space is not Locally $L \mathcal{C}_{\text {-space. }}$

Definition 4.3 A space $X$ is called $w \mathrm{~L}(w \mathcal{C})$. space if for each $w$-Lindelof set in $X$ is $w$-closed.

Definition 4.4 A space $X$ is said to be Locally $w \mathrm{~L}(w \mathcal{C})$-space if each point has a neighborhood which is an $w \mathrm{~L}(w \mathcal{C})$-subspace.

Remark 4.5 Each $w \mathrm{~L}(w \mathcal{C})$-space is Locally $w \mathrm{~L}(w \mathcal{C})$-space.

Theorem 4.6 A space $X_{\text {is }} \mathrm{L}(w \mathcal{C})$-space iff every point in it has clopen set containing $x$ which is $\mathrm{L}(w \mathcal{C})_{\text {-space. }}$

Proof: Let $\mathcal{X}$ be $\mathrm{L}(w \mathcal{C})$-space, so for any $x \in \mathcal{X}$, $X$ it self is clopen that is $\mathrm{L}(w \mathcal{C})$-space.Converse direction, let $\mathcal{D}$ be a Lindelof in $x$ and $x \notin \mathcal{D}$. Choose a clopen $\mathcal{W}_{x \text { containing }} x$ such that $\mathcal{W}_{x}$ is $\mathrm{L}(w \mathcal{C})_{\text {-subspace, if }} \mathcal{D} \cap \mathcal{W}_{x}=\emptyset$, so it is Lindelof also if $\mathcal{D} \cap \mathcal{W}_{x} \neq \emptyset$, it is Lindelof in the subspace $\mathcal{W}_{x}$ by Proposition 2.26, therefore $\mathcal{D} \cap \mathcal{W}_{x}$ is $w_{\text {-closed in }} \mathcal{W}_{x}$ also $w_{\text {-closed in }} X$, hence $\mathcal{W}_{x}-\left(\mathcal{D} \cap \mathcal{W}_{x}\right)=\mathcal{W}_{x}-\mathcal{D}$ is $\mathcal{w}_{\text {-open in }}$

\section{Nadia .A/Haider .J/Rasha .N}

$X$, so $\mathcal{D}$ is $w$-open in $X$, hence $X$ is $\mathrm{L}(w \mathcal{C})$. space.

Corollary 4.7 A space $X$ is $\mathrm{L}(w \mathcal{C})$-space iff every

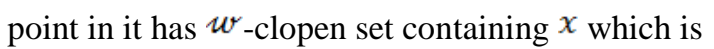
$\mathrm{L}(w \mathcal{C})_{\text {-space. }}$

Proof: From Theorem 4.6, and Remark 2.3, we get the first direction. To prove the second direction, from Theorem 4.6 and Proposition 2.26, a space $X$ is $\mathrm{L}(w \mathcal{C})$-space.

Corollary 4.8 A discrete Locally $\mathrm{L}(w \mathcal{C})$-space is $\mathrm{L}(w \mathcal{C})_{\text {-space. }}$

Theorem 4.9 Any Locally $L(w \mathcal{C})$-space is $w T_{1-}$ space.

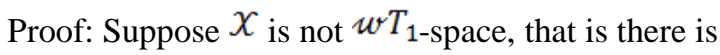

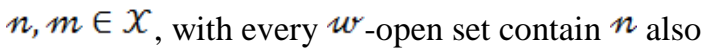

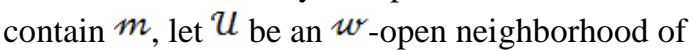

$m$ such that $\left(U, T_{U}\right)$ is $\mathrm{L}(w \mathcal{C})_{\text {-space, since }} X$ is Locally $\mathrm{L}(w \mathcal{C})$-space, so $\left(U, T_{U}\right)$ is $w T_{1 \text {-space, }}$ by Proposition 3.6,_also from Proposition 2.17, $\{n\}$ is $w$-closed in $U$, then $U-\{n\}$ is $w$-open in $U$ and $U$ is $w_{\text {-open in }} X, U-\{n\}$ is $w_{\text {-open in }} X$, by Proposition 2.12, but $m \in \mathcal{U}-\{n\}$ and $n \notin U-\{n\}$, this is contradiction, hence $X$ is $w T_{1-\text { space. }}$

Proposition 4.10 Let $f:(X, T) \rightarrow\left(\mathcal{Y}, T^{\prime}\right)$ be a bijective open function, if $X$ is Locally $L \mathcal{C}_{\text {-space }}$ then $\mathcal{Y}$ is Locally $\mathrm{L}(w \mathcal{C})$-space.

Proof: Let $X$ be a is Locally $\mathrm{LC}_{\text {-space, so for any }}$ $m \in X$, there exists a neighborhood $\mathcal{M}$ of $m$ such that $\mathcal{M}$ is $L \mathcal{C}_{\text {-subspace, but }} \mathcal{M}$ is a neighborhood, then there is open set $\mathcal{H}$ such that $m \in \mathcal{H} \subseteq \mathcal{M}$, so $n=f(m) \in f(\mathcal{H}) \subseteq f(\mathcal{M}) \subseteq \mathcal{Y}$, therefore $\mathcal{Y}$ is Locally $\mathrm{L}(w \mathcal{C})$-space since for any $n \in \mathcal{Y}$, there is an open neighborhood $f(\mathcal{M})$, (since $f$ is open), $\mathcal{M}$

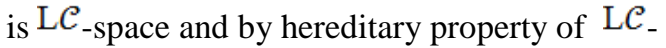
space, we have $\mathcal{H}$ is $\mathrm{L} \mathcal{C}_{\text {-space, then }} f(\mathcal{H})$ is $\mathrm{L} \mathcal{C}$. subspace of $\mathcal{Y}$ from Proposition 2.33, then $f(\mathcal{H})$ is $\mathrm{L}(w \mathcal{C})$-subspace by Remark 3.3 , hence $\mathcal{Y}$ is Locally $\mathrm{L}(w \mathcal{C})$-space. 
Proposition 4.11 Let $f:(X, T) \rightarrow\left(\mathcal{Y}, T^{\prime}\right)$ be a bijectiv $w$-open function. If $\mathcal{H}$ is $w$-Lindelof subset of $\mathcal{Y}$, then $f^{-1}(\mathcal{H})$ is Lindelof subset of $X$.

Proof: Let $\mathcal{H}$ is $w$-Lindelof subset of $\mathcal{Y}$, let $\left\{\mathcal{W}_{\alpha}\right\}_{\alpha \in \Delta}$ be an open cover of $f^{-1}(\mathcal{H})$ in $X$, that is $f^{-1}(\mathcal{H}) \subseteq \mathrm{U}_{\alpha \in \Delta} \mathcal{W}_{\alpha, \text { so }}$ $\mathcal{H}=f\left(f^{-1}(\mathcal{H})\right) \subseteq f\left(\mathrm{U}_{\alpha \in \Delta} \mathcal{W}_{\alpha}\right)=\mathrm{U}_{\alpha \in \Delta} f\left(\mathcal{W}_{\alpha}\right)$, since $f$ is surjective, but $f$ is $w_{\text {-open, so }} f\left(\mathcal{W}_{\alpha}\right)$ is $w$-open for each $\alpha \in \Delta$, and $\mathcal{H}$ is $w$-Lindelof subset of $\mathcal{Y}$, hence $\mathcal{H} \subseteq \mathrm{U}_{\alpha \in \Delta^{\prime}} f\left(\mathcal{W}_{\alpha}\right), \Delta^{4}$ is a countable subset of $\Delta$, also $f^{-1}(\mathcal{H}) \subseteq f^{-1}\left(\mathrm{U}_{\alpha \in \Delta^{\prime}} f\left(\mathcal{W}_{\alpha}\right)\right)=$ $\left.\mathrm{U}_{\alpha \in \Delta^{\prime}} f^{-1} f\left(\mathcal{W}_{\alpha}\right)\right)=\mathrm{U}_{\alpha \in \Delta^{\prime}} \mathcal{W}_{\alpha}$ , therefore $f^{-1}(\mathcal{H})$ is Lindelof in $X$.

Theorem 4.12 Let $f:(X, T) \rightarrow\left(\mathcal{Y}, T^{\prime}\right)$ be a bijectiv $w_{\text {-open function. If }} \mathcal{X}$ is $\mathrm{L} \mathcal{C}_{\text {-space then }} \mathcal{Y}$ is $w \mathrm{~L}(w \mathcal{C})_{\text {-space. }}$

Proof: Let $\mathcal{F}$ be $w$-Lindelof subset of $\mathcal{Y}$, then $f^{-1}(\mathcal{F})$ is Lindelof set in $X$ by Proposition 4.11 , but $X$ is $L \mathcal{C}_{\text {-space, so is }}\left(f^{-1}(\mathcal{F})\right)^{c}$ is open set in $X$, also $f$ is $w_{\text {-open function, hence }}$ $f\left(\left(f^{-1}(\mathcal{F})\right)^{c}\right)=f\left(X-f^{-1}(\mathcal{F})\right.$ $=f(X)-f\left(f^{-1}(\mathcal{F})\right)=\mathcal{Y}-\mathcal{F}=\mathcal{F}^{c}$ is $w_{\text {-open }}$ in $\mathcal{Y}$, so $\mathcal{F}$ is $w$-closed in $\mathcal{Y}$, therefore $\mathcal{Y}$ is $\omega \mathrm{L}(w \mathcal{C})$-space.

Corollary 4.13 Let $f:(X, T) \rightarrow\left(\mathcal{Y}, T^{\prime}\right)$ be a

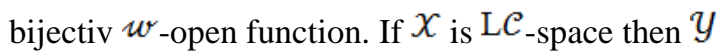
is Locally $w \mathrm{~L}(w \mathcal{C})$-space.

Proof: By Theorem 4.12 and Remark 4.5, we get $\mathcal{Y}$ is Locally $w \mathrm{~L}(w \mathcal{C})_{\text {-space. }}$

Theorem 4.14 Every subspace of Locally L(wC)space is Locally $\mathrm{L}(w \mathcal{C})$-space.

Proof: Let $\mathcal{H}$ be a subspace of a space $\mathcal{X}$, and $c \in \mathcal{P}$, so $c$ has $\mathrm{L}(w \mathcal{C})$ a neighborhood in $X$, hence there is open set $\mathcal{U}_{\text {in }} \mathcal{X}_{\text {such that }} c \in \mathcal{U}$ and $U_{\text {is }} \mathrm{L}(w \mathcal{C})_{\text {-subspace in }} \mathcal{X}$, let $v=\mathcal{H} \cap \mathcal{U}$, then

\section{Nadia .A/Haider .J/Rasha .N}

$\mathcal{V} \subseteq \mathcal{U}$, so $\mathcal{V}$ is $\mathrm{L}(w \mathcal{C})$-subspace from Proposition 3.5 , since $c \in \mathcal{U}$ and $c \in \mathcal{H}$ then $c \in \mathcal{H} \cap \mathcal{U}$, hence $c \in \mathcal{V}=\mathcal{D} \cap \mathcal{U}$, so $\left(\mathcal{H}, T_{\mathcal{H}}\right)$ is Locally $\mathrm{L}(w \mathcal{C})$. subspace of $X$.

\section{References}

[1] AL.Hawary, T. A, AL.Omari, A. , "Between open and $\boldsymbol{\omega}_{\text {-open sets", Question Answers }}$ Gen.Topology, 24 (2006), no. 2,67-78.

[2] Ali, H.J. ,"Certain types of LC-spaces", Thesis submitted to the college of education, ALMustansiriyah University (2005).

[3] AL.Omari, A. and Norani, M. S.,"Regular

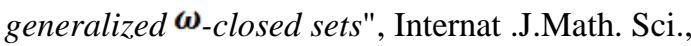
vo 1,11 page (2007).

[4] AL.Omari, A. and Norani, M. S.,"Contra-wcontinuous and Almost Contra-w-continuous", Int. Jour. of Math. And Math. Scie. Vol 2007, 13 (2007), no. 13.

[5] AL.Swidi, L. and Hadi, M. H.

, Characterization of continuity and compactness

with respect to weak form of $\boldsymbol{\omega}_{\text {-open sets", }}$ European journal of scientific research, vol.57, (2011), no.4, 577-582.

[6] Engelking, R. ,"General Topology", (Warszawa, 1977).

[7] Ganster, M. and Jankovic, D. , "On spaces whose Lindelof space are closed", Q \& A in General Topology 7(1989), 141-148.

[8] Ganster, M. ,Kanibir, A. and Reilly, I. ,"Two comments concerning certain topological space”,Indian J. ,Pure Apple. Math. 29 (1998), no.9, 965-967.

[9] Hani, M. H. ,"Weak forms of $\boldsymbol{\omega}_{\text {-open sets and }}$ Decomposition of separation axioms", M. S. C, Thesis, University of Babylon (2011).

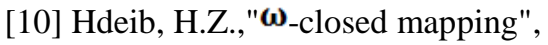
Rev.Colomb.Math.16 (1982) 65-78. 


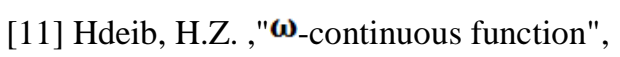
Diraslt,16 , (1989), no.2, 136-142.

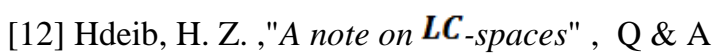
in General Topology 6 (1988), 67-72

[13] Khalaf, A. B. , Darwesh, H. M. , and Kannan, $\mathrm{K}$. ,"Some types of separation axioms in topological spaces", Journal of information and Math. Sci. 28, (2012), no.3,303-326

[14] Levy, R. ,"A non PL-closed space", Q \& A in General Topology 4, (1986), 145-156.

[15] Mukherji, T. K. and Sarkar, M. ,"On a class of almost discrete spaces", Math.Vensik 3 (1979), 459-4754.

[16] Noiri,T. , AL-Omari, A. ,Noorani, M. S.

,Weak forms of $\boldsymbol{\omega}_{-}$-Open sets and decomposition
Nadia .A/Haider .J/Rasha .N

of continuity", E. J. P. A. M 2 (1) (2009), no.3, 384.

[17] Noiri T. and Popa V.,"The unified theory of certain types of generalization of Lindelof spaces" , Demonstratio Math.,43(2010), no11.

[18] Ori, R.G. ,"A note on L-closed spaces", Q \& A in General Topology 4, (1987), 14-143.

[19] Rosas, E. ,Carpintero, C. ,Salas, M. ,Sanabrla, J. and Vasquez, L., "Almost w-continuous functions defined by w-open sets due to Arhangelskii", CUBO A Mathematical journal ,19 (2017), no.1, 115 .

[20] Zaynab, A. M. ,"On $\boldsymbol{\omega}_{\text {-proper mappings", }}$ M.S.C.Thesis University of Kufa (2014). 


$$
\text { فضاعات_L(wC) L والصيغ الضعبفه لها }
$$

$$
\begin{aligned}
& \text { ناديه علي ناظم' } \\
& \text { حيدر جبر علي' } \\
& \text { رشـا ناصر مجيدَّ }
\end{aligned}
$$

Nadia .A/Haider .J/Rasha .N 